\title{
Pharmacy Electronic Records and Patient Clustering: Exploring New Ways to Increase the Provision of Tailored Pharmaceutical Services
}

\author{
Lígia REIS $^{\mathrm{a}, \mathrm{b}}$, Miguel MONTEIRO ${ }^{\mathrm{b}}$, Luís LOURENÇO ${ }^{\mathrm{b}}$ and João GREGÓRIO ${ }^{\mathrm{a}, 1}$ \\ ${ }^{\mathrm{a}}$ CBIOS - Universidade Lusófona's Research Center for Biosciences \& Health \\ Technologies, Lisbon, Portugal \\ ${ }^{\mathrm{b}}$ Farmácia Central do Cacém, Lisbon, Portugal
}

\begin{abstract}
Patients' electronic records in community pharmacy are an untapped resource to uncover new ways of providing healthcare services. In this paper, we present a preliminary work, where we explore this resource, aiming to identify patients' clusters that will help to define a future algorithm. This algorithm will then enable community pharmacists to provide tailored pharmaceutical interventions according to patient's risk assessment and needs. In this way, this work will provide a way to overcome known barriers for community pharmacists' provision of services and integration in the health system, while also contributing to support a better care for chronic patients.
\end{abstract}

Keywords. Electronic Health Records; Algorithms; Pharmaceutical Services

\section{Introduction}

Community pharmacy collects patient information continuously, creating a permanently updated medication record. This information is kept and stored but remains untapped.[1]

Algorithms, queries, and knowledge-based systems are some approaches to screen electronic patient records stored in databases and support pharmacist medication reviews.[2] However, the definition of an algorithm tailored to the Portuguese pharmacies' information system is lacking. Thus, the aim of this study was to perform a medication review on patient's medication records to identify clusters that will enable the definition of an algorithm to tailor pharmacy professional interventions.

\section{Methods}

A retrospective observational study was performed. Records were included if patients had a continuous medication dispensing history between June 2017 - July 2018 and used

1 Corresponding author, João Gregório, CBIOS, Universidade Lusófona's Research Center for Biosciences \& Health Technologies, Campo Grande 376, 1749-024 Lisboa, Portugal; E-mail: joao.gregorio@ulusofona.pt. 
two or more chronic medications. Statistical analysis used a two-step cluster analysis to identify common characteristics among patients.

\section{Results}

The final sample of electronic records eligible for simple medication review included 55 patients. The variables included in the two-step cluster analysis were severity degree of interactions, severity degree of contraindications, Beers criteria, number of drugs used and medical condition with measurable biomarkers. Four clusters and one outlier patient were identified. Each cluster was assigned a different level of pharmaceutical intervention.

\section{Discussion}

The results showed that all of the patients in this sample could benefit from professional interventions beyond the basic medicine dispensing service. Two clusters, representing $36.3 \%$ of patients, could benefit from an advanced medication review, while one cluster of $30.9 \%$ patients would only need to measure their biomarkers in order to assess the effectiveness of the therapy. Also, the medication review identified one high risk patient, requiring an urgent medication review. Therefore, this non-clinical review was successful in exposing five different levels of pharmaceutical services' needs, with the purpose of highlighting more complex issues for other type of medication reviews or pharmaceutical services.

In order to help pharmacists in the provision of medication reviews without increasing workload, the full automation of this initial medication review is desirable. A computerized system based on an algorithm might support a less time-consuming, continuous, and reproducible medication review.

\section{Conclusions}

To provide more and better professional services, community pharmacists need tools and systems that can analyze this information, processing data easily, quickly, and continuously. The statistical analysis carried out in this study allowed the grouping of patients into clusters, enabling their prioritization and subsequent suggestion of pharmaceutical interventions according to their health needs. This clustering will provide the foundation for the design of a criteria-based algorithm likely to be automated, which will assist community pharmacists in providing better care for chronic patients.

\section{References}

[1] Jackson S, Peterson G. My Health Record: a community pharmacy perspective. Aust Prescr. 2019;42(2):46-47. doi:10.18773/austprescr.2019.009.

[2] Sacro K, Smith M, Swedberg C, Lee YJ, Hunt M, Mulrooney M. PharmValCalc: A calculator tool to forecast population health pharmacist impact. Res Social Adm Pharm. 2020;16(9):1183-1191. doi:10.1016/j.sapharm.2019.12.012. 